doi: $10.13108 / 2014-6-1-12$

UDC 517.9

\title{
ON ANALOGUES OF THIRD ORDER BESSEL FUNCTION
}

\section{F.KH. BAICHOROVA}

\begin{abstract}
We consider eigenfunctions of differential operators semi-invariant with respect to to the group of translations. We obtain a solvability condition in terms of primitive functions and show a connection of this condition with the theory of commutative rings of differential operators.
\end{abstract}

Keywords: generalized Bessel functions, Darboux transformations.

Mathematics Subject Classification: 34L05

\section{INTRODUCTION}

We consider the eigenvalue problem for differential operators in the following special class (cf. [1])

$$
A=e^{N t} a\left(D_{t}\right)=e^{N t} \prod_{j=1}^{N}\left(D_{t}+\alpha_{j}\right) .
$$

Here $a\left(D_{t}\right)$ is arbitrary polynomial with constant coefficients of order $N$ depending on the symbol $D_{t}$. After the change of independent variable

$$
x=-e^{-t}, \quad D_{x}=e^{t} D_{t}, \quad D_{t}=-x D_{x},
$$

operator (1) becomes Euler operator (cf. [2])

$$
\widetilde{A}=\frac{1}{(-x)^{N}} \prod_{j=1}^{N}\left(x D_{x}-\alpha_{j}\right),
$$

and as $N=2$ we get

$$
\widetilde{A}=\frac{1}{x^{2}}\left(x D_{x}-\alpha_{1}\right)\left(x D_{x}-\alpha_{2}\right)=D_{x}^{2}+\frac{\alpha}{x} D_{x}+\frac{\beta}{x^{2}} .
$$

In other words, as $N=2$, the considered eigenvalue problem $A \psi=\lambda \psi$ is reduced to the Bessel equation

$$
y^{\prime \prime}+\frac{1}{x} y^{\prime}=\frac{x^{2}+n^{2}}{x^{2}} y
$$

while as $N=3$, it reduces to a simplest generalization of equation (5).

Operators (1) are semi-invariant with respect to the translation $t \rightarrow t+$ const. By means of the translation, without loss of generality we let $\lambda=1$ in equation $A \psi=\lambda \psi$. We notice then that the operation of conjugation with exponent is equivalent to the change $D_{t} \rightarrow D_{t}+$ const for these operators. Indeed,

$$
e^{-k t} \circ D_{t} \circ e^{k t}=D_{t}+k \Rightarrow e^{-k t} \circ e^{N t} a\left(D_{t}\right) \circ e^{k t}=e^{N t} a\left(D_{t}+k\right) .
$$

F.Kh. Baichorova, On the analogues of the Bessel function of the third order.

(C) BAICHOROVA F.KH. 2014.

Submitted November 26, 2013. 
Thus, as $N=3$, we choose one of the zeroes of operator (1) being zero and we reduce the eigenvalue equation to the form

$$
e^{3 t} D_{t}\left(D_{t}+\alpha_{2}\right)\left(D_{t}+\alpha_{3}\right) \psi=\psi \Leftrightarrow \psi^{\prime \prime \prime}+\frac{\alpha}{x} \psi^{\prime \prime}+\frac{\beta}{x^{2}} \psi^{\prime}=\psi
$$

where $\alpha=3-\alpha_{2}-\alpha_{3}, \beta=1-\alpha_{2}-\alpha_{3}+\alpha_{2} \alpha_{3}$.

It is easy to see that the point $x=0$ is a regular critical point for equation (7). As it is known, in a neighborhood of a regular critical point for this equation there exists a fundamental system of three solutions represented by converging power series (see [3])

$$
\psi=x^{s}\left(\widehat{C}_{0}+\widehat{C}_{1} x+\widehat{C}_{2} x^{2}+\ldots\right)
$$

Generally speaking, in this formula the logarithm appears and in the case of "multiple" roots the solution is to be sought as

$$
\psi=x^{s}\left[\varphi_{1}(x) \log (x)+\varphi_{2}(x)\right]
$$

where $\varphi_{1}(x), \varphi_{2}(x)$ are analytic at zero functions. The sufficient condition of absence of logarithm is that numbers $\alpha_{2}, \alpha_{3}$ to be non-integer, this case is considered below (see (14)).

Substituting expansion of $\psi$ and its derivatives in equation (7) and equating the coefficients at the like powers of $x$ to zero, we obtain equation

$$
s[(s-1)(s-2)+\alpha(s-1)+\beta]=0,
$$

which determines exponent $s$. This equation is called determining equation. Its solutions are $0, \alpha_{2}, \alpha_{3}$. The general case is a non-integer $s$.

Example 1. As $\alpha_{2}=1, \alpha_{3}=2$ eigenvalue equation (7) is reduced to the equation

$$
\left(e^{t} D_{t}\right)^{3} \widehat{\psi}=D_{x}^{3} \widehat{\psi} \quad \Leftrightarrow \quad \widehat{\psi}^{\prime \prime \prime}=\widehat{\psi}
$$

whose fundamental system of solutions is

$$
\widehat{\psi}_{1}=e^{x}, \widehat{\psi}_{2}=e^{a x}, \widehat{\psi}_{3}=e^{b x}
$$

where $a=-\frac{1}{2}(1+i \sqrt{3}), b=\bar{a}$.

The main result of the work is the following statement.

Eigenvalue problem for the third order operator $\widetilde{A}=\frac{1}{x^{3}}\left(x D_{x}-\alpha_{1}\right)\left(x D_{x}-\alpha_{2}\right)\left(x D_{x}-\alpha_{3}\right)$ has three solutions expressed in terms of primitive functions, namely,

$$
\psi_{j}=e^{\alpha_{j} x} \sum_{k=0}^{n} C_{k} x^{k}
$$

if the condition

$$
\left(0, \alpha_{2}, \alpha_{3}\right) \equiv(0,1,2) \quad(\bmod \quad 3)
$$

holds true.

In the case of second order Bessel equation, the condition similar (9) is written as

$$
\left(0, \alpha_{2}\right) \equiv(0,1) \quad(\bmod \quad 2)
$$

that is equivalent to half-integer $n$ in equation (5).

It was shown in work [4] that integer $n$ in equation (5) correspond to commutative rings of forth and sixth order differential operators (1). 


\section{EIGENFUnCTIONS}

While finding the coefficients of power in $x$ expansion (8) for solution, it is convenient to rewrite the differential equation in terms of the powers of Euler operator $x D_{x}=\theta$. For instance, differential operator

$$
P=x(1-x) \partial^{2}-(a+b+1) x \partial+c \partial-a b,
$$

corresponding to the hypergeometric Gauss equation, can be rewritten as (cf. [5])

$$
x \cdot P=\theta(\theta+c-1)-x(\theta+a)(a+b) .
$$

by employing Euler operator $\theta=x \partial$.

In the general case of third order operator

$$
A=e^{3 t} D_{t}\left(D_{t}+\alpha_{2}\right)\left(D_{t}+\alpha_{3}\right)=\frac{1}{x^{3}} \theta\left(\theta-\alpha_{2}\right)\left(\theta-\alpha_{3}\right)=\frac{1}{x^{3}} a(\theta),
$$

it is more convenient to see an eigenfunction as the product of a polynomial and an exponential

$$
\psi=e^{x} \sum_{k=0}^{n} C_{k} x^{k}, \quad C_{0}=\text { const. }
$$

At that, the coefficients $\widehat{C}_{k}$ and $C_{k}$ of expansions (8) and $(13)$ for an eigenfunction are related by the formulae

$$
\widehat{C}_{k}=\sum_{i+j=k} \frac{C_{i}}{j !}=\frac{C_{0}}{k !}+\ldots C_{k}, \quad k>0 .
$$

Moreover, the following statement holds true. In the general case

$$
\widehat{C}_{0}=1, \quad \widehat{C}_{1}=0, \quad \widehat{C}_{2}=0, \quad \widehat{C}_{3}=\frac{1}{a(3)}, \ldots, \quad \widehat{C}_{k}=\frac{\widehat{C}_{k-3}}{a(k)}
$$

and solutions to the problem $A \psi=\psi$ are entire functions.

Indeed, suppose that a solution to the problem $A \psi=\psi$ is represented by power series (8), where $\widehat{C}_{k}$ are determined by (14).

Let us show that function (8) is entire. In order to do it, let us find the convergence domain for series (8) by means of D'Alambert test

$$
\begin{aligned}
\lim _{k \rightarrow \infty}\left|\frac{\widehat{C}_{3 k+3}}{\widehat{C}_{3 k}}\right| & =\lim _{k \rightarrow \infty}\left|\frac{x^{3 k+3} \prod_{k}\left(3 k\left[(3 k)^{2}+3 k b_{1}+b_{2}\right]\right)}{x^{3 k} \prod_{k}\left[(3 k+3)\left((3 k+3)^{2}+(3 k+3) b_{1}+b_{2}\right)\right]}\right| \\
& =\lim _{k \rightarrow \infty}\left|\frac{x^{3}}{(3 k+3)\left((3 k+3)^{2}+(3 k+3) b_{1}+b_{2}\right)}\right| \\
& =\left|x^{3}\right| \lim _{k \rightarrow \infty}\left|\frac{1}{(3 k+3)\left((3 k+3)^{2}+(3 k+3) b_{1}+b_{2}\right)}\right|=0 .
\end{aligned}
$$

The radius of the convergence circle equals $\infty$ and series (8) converges absolutely for each $x$. An arbitrary polynomial $a(\theta)$ in (12) satisfies the following relations (cf. [5]):

$$
\begin{aligned}
& a(\theta) \bullet x^{\alpha}=a(\alpha) \cdot x^{\alpha}, \\
& a(\theta)\left(x^{i}\right)=x^{i} \cdot a(\theta+i), \\
& a(\theta)\left(e^{x} x^{k}\right)=x^{k} a(\theta+k)\left(e^{x}\right) .
\end{aligned}
$$

Applying these formulae to $a(\theta)=\theta^{3}+b_{1} \theta^{2}+b_{2} \theta$, where $b_{1}=-\alpha_{2}-\alpha_{3}, b_{2}=\alpha_{2} \alpha_{3}$, we obtain $a(\theta+k)=\theta^{3}+\left(b_{1}+3 k\right) \theta^{2}+\left(3 k^{2}+2 b_{1} k+b_{2}\right) \theta+a(k), \quad a(k)=k^{3}+b_{1} k^{2}+b_{2} k, \quad k=0,1,2, \ldots$ 
Hence,

$$
a(\theta+k)\left(e^{x}\right)=e^{x}\left(x^{3}+\left(b_{1}+3 k+3\right) x^{2}+b_{2}(k) x+a(k)\right), \text { where } \quad b(k)=a(k+1)-a(k) .
$$

Let us find coefficients $C_{k}$ of series 113 by equation $A \psi=\psi$ :

$$
A \psi=\frac{1}{x^{3}} a(\theta)\left(e^{x} \sum_{k=0}^{n} C_{k} x^{k}\right)=e^{x} \sum_{k=0}^{n} C_{k} x^{k} .
$$

We equate the coefficients at the like powers of $x$ :

$$
\begin{aligned}
& a(1)\left(C_{0}+C_{1}\right)=0, \\
& (3+a) C_{0}+b(1) C_{1}+a(2) C_{2}=0, \\
& C_{0}+(6+a) C_{1}+b(2) C_{2}+a(3) C_{3}=C_{0}, \\
& C_{1}+(9+a) C_{2}+b(3) C_{3}+a(4) C_{4}=C_{1}, \\
& C_{2}+(12+a) C_{3}+b(4) C_{4}+a(5) C_{5}=C_{2},
\end{aligned}
$$

or, taking into consideration (19),

$$
\left\{\begin{array}{c}
a(1)\left(C_{0}+C_{1}\right)=0 \\
(3+a) C_{0}+[a(2)-a(1)] C_{1}+a(2) C_{2}=0 \\
(6+a) C_{1}+[a(3)-a(2)] C_{2}+a(3) C_{3}=0 \\
\left.(9+a) C_{2}+[a(4)-a(3))\right] C_{3}+a(4) C_{4}=0 \\
(12+a) C_{3}+[a(5)-a(4)] C_{4}+a(5) C_{5}=0 \\
\cdots
\end{array}\right.
$$

By the obtained system one can deduce recurrent relation for the coefficients $C_{k}$ :

$$
C_{k+1}=-\frac{C_{k-1}(a+3 k)+[a(k+1)-a(k)] C_{k}}{a(k+1)} .
$$

\section{BREAK CONDITIONS}

Consider the case when the solutions for the eigenvalue problem

$$
A \psi=\psi
$$

for operator 12 are the functions

$$
\psi=e^{x}\left(C_{0}+C_{1} x+C_{2} x^{2}+C_{3} x^{3}\right) .
$$

Let us solve above system (20) for the first, second and third power of the polynomial in (13).

For the first power we vanish all the coefficients starting from $C_{2}$, then system $(20)$ casts into the form

$$
\left\{\begin{array}{l}
a(1)\left(C_{0}+C_{1}\right)=0, \\
(3+a) C_{0}+[a(2)-a(1)] C_{1}=0 \\
(6+a) C_{1}=0 .
\end{array}\right.
$$

It splits into other two:

$$
\left\{\begin{array}{l}
a(1)=0 \\
(3+a) C_{0}+[a(2)-a(1)] C_{1}=0 \\
(6+a) C_{1}=0
\end{array}\right.
$$


and

$$
\left\{\begin{array}{l}
C_{0}+C_{1}=0 \\
(3+a) C_{0}+[a(2)-a(1)] C_{1}=0 \\
(6+a) C_{1}=0
\end{array}\right.
$$

Solving these systems, we obtain

(1) $b_{1}=-6, b_{2}=5, C_{1}=-\frac{1}{2} C_{0}$, the eigenfunction for operator $A$ is $\psi_{1}=e^{x}\left(C_{0}-\frac{1}{2} C_{0} x\right)$.

Or

(2) $b_{1}=-6, b_{2}=8, C_{1}=-C_{0} ; \psi_{1}=e^{x}\left(C_{0}-C_{0} x\right)$.

In the same way, for the second power we vanish all the coefficients starting from $C_{3}$, while for the third power we have $C_{i}=0, i=4,5 \ldots$

The associated solutions are

(3) $b_{1}=-9, b_{2}=8, C_{1}=-\frac{3}{5} C_{0}, C_{2}=\frac{C_{0}}{10} ; \psi_{1}=e^{x}\left(C_{0}-\frac{3}{5} C_{0} x+\frac{1}{10} C_{0} x^{2}\right)$,

(4) $b_{1}=-9, b_{2}=20, C_{1}=-C_{0}, C_{2}=\frac{1}{2} C_{0} ; \psi_{1}=e^{x}\left(C_{0}-C_{0} x+\frac{1}{2} C_{0} x^{2}\right)$,

(5) $b_{1}=-9, b_{2}=14, C_{1}=-C_{0}, C_{2}=\frac{1}{4} C_{0} ; \psi_{1}=e^{x}\left(C_{0}-C_{0} x+\frac{1}{4} C_{0} x^{2}\right)$,

(6) $b_{1}=-12, b_{2}=11, C_{1}=-\frac{13}{20} C_{0}, C_{2}=\frac{3}{20} C_{0}, C_{3}=-\frac{1}{80} C_{0} x^{3}$,

$$
\psi_{1}=e^{x}\left(C_{0}-\frac{13}{20} C_{0} x+\frac{3}{20} C_{0} x^{2}-\frac{1}{80} C_{0} x^{3}\right),
$$

(7) $b_{1}=-12, b_{2}=35, C_{1}=-C_{0}, C_{2}=\frac{C_{0}}{2}, C_{3}=-\frac{C_{0}}{8}$;

$$
\psi_{1}=e^{x}\left(C_{0}-C_{0} x+\frac{C_{0}}{2} x^{2}-\frac{1}{8} C_{0} x^{3}\right),
$$

(8) $b_{1}=-12, b_{2}=32, C_{1}=-C_{0}, C_{2}=\frac{C_{0}}{2}, C_{3}=-\frac{C_{0}}{10}$;

$$
\psi_{1}=e^{x}\left(C_{0}-C_{0} x+\frac{C_{0}}{2} x^{2}-\frac{1}{10} C_{0} x^{3}\right),
$$

(9) $b_{1}=-12, b_{2}=20, C_{1}=-C_{0}, C_{2}=\frac{9 C_{0}}{28}, C_{3}=-\frac{C_{0}}{28}$;

$$
\psi_{1}=e^{x}\left(C_{0}-C_{0} x+\frac{9 C_{0}}{28} x^{2}-\frac{1}{28} C_{0} x^{3}\right) .
$$

In order to construct two other solutions, we shall consider the right operator of Darboux transformation $R$ relating operator $A$ of the form (1) and the operator $A_{0}=\left(e^{t} D_{t}\right)^{3}=D_{x}^{3}$ in Example 1. It was shown in work [1] that this operator exists, if condition (9) holds true. It can be checked by straightforward calculations that this condition is satisfied for the above cases (1)-(9).

At that,

$$
A R=R A_{0} \Rightarrow A \psi=\psi \quad \text { as } \quad \psi=R \widehat{\psi},
$$

and operator $R$ maps eigenfunctions of operator $A_{0}$ the eigenfunctions of operator $A$. For instance, for the operator $A=e^{3 t} D_{t}\left(D_{t}+1\right)\left(D_{t}+5\right)$ we have

$$
\psi_{2}=R \widehat{\psi}_{2}=\left(D_{t}+2\right) e^{\alpha x}=\left(-x D_{x}+2\right) e^{\alpha x}=e^{\alpha x}(2-x \alpha)
$$

and

$$
\psi_{3}=R \widehat{\psi}_{3}=\left(-x D_{x}+2\right) e^{\beta x}=e^{\beta x}(2-x \beta) .
$$

In the same way one can find eigenfunctions for each of cases (1)-(9).

The above formulae for $\psi_{1}$ allow one to find explicitly operator $R$ (cf. [1]) for each of cases (1)-(9). For instance, let us find the operator $R=\left(D_{t}+\gamma_{1}\right)\left(D_{t}+\gamma_{2}\right)$ by eigenfunction (3), 
rewriting $R$ in terms of $x$ :

$$
\begin{aligned}
& R=\left(-x D_{x}+\gamma_{1}\right)\left(-x D_{x}+\gamma_{2}\right)=x^{2} D_{x}+\left(1-\gamma_{1}-\gamma_{2}\right) x D_{x}+\gamma_{1} \gamma_{2}, \\
& R e^{x}=e^{x}\left(x^{2}+\left(1-\gamma_{1}-\gamma_{2}\right) x+\gamma_{1} \gamma_{2}\right)=e^{x}\left(10-6 x+x^{2}\right)=\psi_{1},
\end{aligned}
$$

that yields

$$
\left\{\begin{array}{l}
1-\gamma_{1}-\gamma_{2}=-6 \\
\gamma_{1} \gamma_{1}=10
\end{array}\right.
$$

or $\gamma_{1}=2, \gamma_{1}=5$ and $R=\left(D_{t}+2\right)\left(D_{t}+5\right)$.

In the next table we provide operators $A$ and related transformation operators $R$ :

\begin{tabular}{|l|l|l|}
\hline No. & \multicolumn{1}{|c|}{$A$} & \multicolumn{1}{|c|}{$R$} \\
\hline \hline$(1)$ & $e^{3 t} D_{t}\left(D_{t}+1\right)\left(D_{t}+5\right)$ & $D_{t}+2$ \\
\hline \hline$(2)$ & $e^{3 t} D_{t}\left(D_{t}+2\right)\left(D_{t}+4\right)$ & $D_{t}+1$ \\
\hline \hline$(3)$ & $e^{3 t} D_{t}\left(D_{t}+1\right)\left(D_{t}+8\right)$ & $\left(D_{t}+2\right)\left(D_{t}+5\right)$ \\
\hline \hline$(4)$ & $e^{3 t} D_{t}\left(D_{t}+4\right)\left(D_{t}+5\right)$ & $\left(D_{t}+1\right)\left(D_{t}+2\right)$ \\
\hline \hline$(5)$ & $e^{3 t} D_{t}\left(D_{t}+2\right)\left(D_{t}+7\right)$ & $\left(D_{t}+1\right)\left(D_{t}+4\right)$ \\
\hline \hline$(6)$ & $e^{3 t} D_{t}\left(D_{t}+1\right)\left(D_{t}+11\right)$ & $\left(D_{t}+2\right)\left(D_{t}+5\right)\left(D_{t}+8\right)$ \\
\hline \hline$(7)$ & $e^{3 t} D_{t}\left(D_{t}+5\right)\left(D_{t}+7\right)$ & $\left(D_{t}+1\right)\left(D_{t}+2\right)\left(D_{t}+4\right)$ \\
\hline \hline$(8)$ & $e^{3 t} D_{t}\left(D_{t}+4\right)\left(D_{t}+8\right)$ & $\left(D_{t}+1\right)\left(D_{t}+2\right)\left(D_{t}+5\right)$ \\
\hline \hline$(9)$ & $e^{3 t} D_{t}\left(D_{t}+2\right)\left(D_{t}+10\right)$ & $\left(D_{t}+1\right)\left(D_{t}+4\right)\left(D_{t}+7\right)$ \\
\hline
\end{tabular}

\section{BIBLIOGRAPHY}

1. A.B. Shabat, Z.S. Élkanova, A.B. Urusova. Two-sided Darboux transformations. Teor. matem. fiz. 173:2, 207-218 (2012). [Theor. Math. Phys. 173:2, 1507-1517 (2012).]

2. Yu.S. Il'yashenko, S.Yu. Yakovenko. Analytic theory of differential equations. MCCME, Moscow (2013). (in Russian).

3. E.L. Ains. Ordinary differential equations, NTIU, Kharkov (1939). (in Russian).

4. F.Kh. Baichorova, Z.S. Elkanova. Commuting differential operators of order 4 and 6 . Ufimskij matem. zhur. 5:3, 12-20 (2013). [Ufa Math. J. 5:3, 11-19 (2013).]

5. M. Saito, B. Sturmfels, N. Takayama. Grobner Deformations of Hypergeometric Differential Equations. Springer-Verlag, Berlin (2000).

Fatima Khasanovna Baichorova,

Aliyev Karachay-Cherkess State University,

Lenin str., 29

4369202, Karachaevsk, Karachay-Cherkess Republic, Russia

E-mail: fatima-kchgu@yandex.ru 